\title{
Second Order Volterra Inverses for Compensation of Loudspeaker Nonlinearity
}

\author{
Hans Schurer student member, IEEE, Cornelis H. Slump member, IEEE and Otto E. Herrmann member, IEEE \\ University of Twente, Dept. of Electrical Eng., Laboratory for Network Theory and VLSI Design, \\ P.O. Box 217, 7500 AE Enschede, The Netherlands, Tel/Fax: +31 [53] 892673/334701, \\ E-mail: hans@nt.el.utwente.nl
}

\begin{abstract}
High quality sound reproduction by loudspeakers is increasingly problematic if the dimensions of the loudspeaker decrease. To produce enough power, large diaphragm excursions are needed which give rise to significant distortions especially at very low frequencies. Instead of improving the mechanical construction of the transducer we apply a feedforward nonlinear digital inverse circuit. Results of two $2^{\text {nd }}$ order Volterra compensators show a significant reduction of the second order harmonics, leaving higher order distortions unchanged. The structure of the realization influences the performance considerably. Two realization structures are considered, and the error caused by the differentiators in the output of the compensators are compared. Both algorithms are implemented in real-time on a Digital Signal Processor (DSP) for on-line testing with the transducer.
\end{abstract}

\section{Introduction}

Because audio reproduction elements tend to decrease in size there is a search for smaller loudspeakers as well. Especially for low frequency radiators this minimization of dimensions has physical limits. To obtain a high quality response for low frequencies, excessive diaphragm excursions are needed which generate high distortions. A method to improve the transfer behavior of electro-acoustical transducers is by changing the magnetic or mechanical design. Disadvantage of this kind of changes is a more complex assembly and thus an increasing prize of the transducer.

In this paper we improve the behavior of the loudspeaker by means of an algorithm implemented on a DSP. With the decreasing prizes of digital processing hardware this is a realistic approach to the mentioned design problem. The compensators are realized as feedforward controllers. Therefore an additional sensor like a microphone or an accelerometer is superfluous. Such sensors make the system more expensive, and their quality determines the effectiveness of the controller. Moreover, in contrast with feedback solutions, there is no loss of sound pressure by application of a feedforward controller.

\section{Modeling}

In the electrodynamic loudspeaker sound waves are produced by a diaphragm driven by an alternating current through a voice coil which is positioned in a permanent magnetic field. Most nonlinearities of the transducer are due to the displacement $x$ of the diaphragm. Three nonlinearities are found to be of major influence [1]:

- the transduction between electric and mechanic domain, also known as the force factor: $B l(x)$

- the stiffness of the spider suspension: $1 / C_{m}(x)$

- the self inductance of the voice coil: $L_{e}(x)$

The dynamical behavior of the electrodynamic loudspeak- er driven by an input voltage $u_{e}$ is given by two coupled nonlinear differential equations [2]:

$$
\begin{aligned}
& u_{e}=R_{e} i+\frac{d\left(L_{e}(x) i\right)}{d t}+B l(x) \dot{x} \\
& B l(x) i=m_{t} \ddot{x}+R_{m} \dot{x}+\frac{1}{C_{m}(x)} x
\end{aligned}
$$

Where Eq.(1) describes the electrical port of the transducer with input current $i$ and voice coil resistance $R_{\ell}$. The mechanical part is given by Eq.(2) which is a simple damped $\left(R_{m}\right)$ mass $\left(m_{t}\right)$-spring $\left(C_{m}(x)\right)$ system driven by the force $B l(x) i$.

The displacement dependent parameters $L_{\ell}(x), B l(x)$ and $C_{m}(x)$ are described by a Taylor series expansion, truncated after the second term:

$$
\begin{aligned}
& L_{e}(x)=L_{e 0}+l_{1} x \\
& B l(x)=B l_{0}+b_{1} x \\
& C_{m}(x)=C_{m 0}+c_{1} x
\end{aligned}
$$

In this way we first model, and compensate, the produced second order harmonic and intermodulation distortion. The total nonlinear differential equation is obtained from substitution of Eq.(2) into Eq.(1) using Eq.(3).

Parameters, linear and nonlinear, are determined by optimization on input impedance and sound pressure response measurements. Linear parameters are optimized using a least squares fit on input impedance measurements and nonlinear model parameters $\left(l_{l}, b_{l}\right.$ and $\left.c_{l}\right)$ are optimized using a Simplex [3] search method on the nonlinear model.

\section{Volterra inverses 3.1 Theory}

From the nonlinear differential equations we derive a nonlinear inverse filter to eliminate the nonlinear behavior, based on a Volterra series expansion of the transducer. In 
general the output $y(t)$ of a nonlinear system, characterized by a continuous time Volterra series, is given by:

$$
\begin{aligned}
y(t)= & h_{0}+\int_{-\infty}^{+\infty} h_{1}(t) x(t-\tau) d \tau+ \\
& \int_{-\infty}^{+\infty} \int_{-\infty}^{+\infty} h_{2}\left(\tau_{1}, \tau_{2}\right) x\left(t-\tau_{1}\right) x\left(t-\tau_{2}\right) d \tau_{1} d \tau_{2}+\ldots
\end{aligned}
$$

where $x(t)$ is the system input and $h_{n}\left(\tau_{1}, \ldots, \tau_{n}\right)$ are the generalized impulse responses, also called kernels. Similar to linear systems we can determine, using the multi-dimensional Laplace transform, the response in the frequency domain $(s=\sigma+j \omega$ is the complex frequency variable):

$Y(s)=H_{1}(s) X(s)+\Gamma\left\{H_{2}\left(s_{1}, s_{2}\right) X\left(s_{1}\right) X\left(s_{2}\right)\right\}+\ldots$

where the capital letters denote the Laplace transformed versions of their small letter counterparts and $\Gamma / \int$ the contraction operator. The system response is now determined by a summation of all kernel responses, i.e. the Volterra series can be seen as a Taylor series with memory [4]. From this nonlinear system representation we determine a compensator which eliminates the kernel $h_{2}\left(\tau_{1}, \tau_{2}\right)$. This compensator is given by:

$K_{2}\left(s_{1}, s_{2}\right)=\frac{-H_{2}\left(s_{1}, s_{2}\right)}{H_{1}\left(s_{1}+s_{2}\right)}$

The frequency domain versions of the linear kernel $H_{l}$ and nonlinear kernel $H_{2}$ are derived from the nonlinear differential equation driven with a two tone excitation.

\subsection{Realization structures}

From the nonlinear model of the loudspeaker given by Eqs. (1) and (2) the second order Volterra compensator kernel in the frequency domain is found to be [1]:

$$
\begin{gathered}
K_{2}\left(s_{1}, s_{2}\right)=H_{x}\left(s_{1}\right) H_{x}\left(s_{2}\right)\left\{a+b\left(s_{1}+s_{2}\right)+c\left(s_{1}+s_{2}\right)^{2}\right. \\
\left.+d\left(s_{1}+s_{2}\right)^{3}-s_{1} s_{2}\left[e+f\left(s_{1}+s_{2}\right)\right]\right\}
\end{gathered}
$$

with $H_{x}(s)$ the linear transfer function from input voltage to displacement $x$ of the cone, and $a$ through $f$ constant parameters formed by the linear and nonlinear parameters.

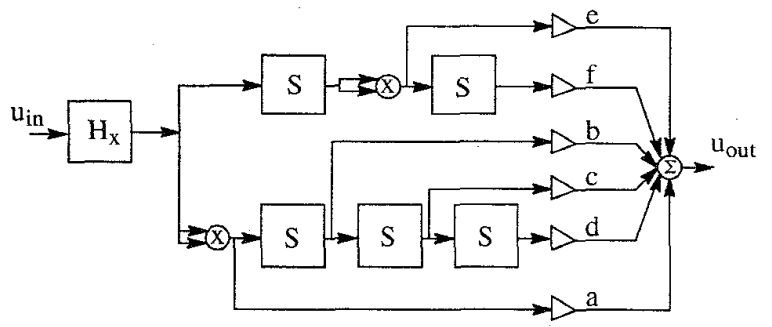

Figure 1: Second order compensator-1 according to Eq.(7).
Direct realization of this compensator yields an inefficient implementation which needs five differentiators as depicted in Figure 1 (the $S$-blocks depict the differentiators). A more efficient form is found if we first rewrite Eq.(7) into:

$$
\begin{aligned}
K_{2}\left(s_{1}, s_{2}\right)= & \frac{1}{2} H_{x}\left(s_{1}\right) H_{x}\left(s_{2}\right)\left\{E\left(s_{1}^{3}+s_{2}^{3}\right)+G\left(s_{1}^{2} s_{2}+s_{1} s_{2}^{2}\right)\right. \\
& +D\left(s_{1}^{2}+s_{2}^{2}\right)+C\left(s_{1}+s_{2}\right)+2 F s_{1} s_{2}+2 B+ \\
& \left.+A\left\{\frac{1}{H_{x}\left(s_{1}\right)}+\frac{1}{H_{x}\left(s_{2}\right)}\right\}\right\}
\end{aligned}
$$

with $A$ through $F$ again constant parameters containing linear and nonlinear parameters of the loudspeaker. Normally, a Volterra kernel of the form $K_{g}\left(s_{1}, s_{2}\right)$ $=K_{a}\left(s_{I}\right) K_{b}\left(s_{2}\right) K_{\mathcal{C}}\left(s_{I}+s_{2}\right)$, is synthesized using three linear filters and one multiplier [4]. However, due to the structure of Eq.(8) it is possible to obtain a more efficient realization. Using several simplification techniques we obtain a second order compensator as depicted in Figure 2, containing only three differentiators.

\subsection{Error analysis}

The realizations in Figure 1 and 2 consist of a linear filter $H_{x}(s)$, adders and multipliers, elements which can be easily rcalized digitally. Linear filter $H_{x}(z)$ is obtained from its continuous frequency domain counterpart by means of the bilinear transformation.

The most important elements are the differentiators which, despite of ongoing research, are difficult to realize. We choose to realize them as IIR filters based on the Simpson integration rule [5]. This yields a very efficient realization given by:

$S(z)=\frac{3\left(1-z^{-2}\right)}{T 3.7321\left(1+0.5358 z^{-1}+0.0718 z^{-2}\right)}$

with $T$ the sampling period. This differentiator has a very small error and nearly linear phase in the low frequency

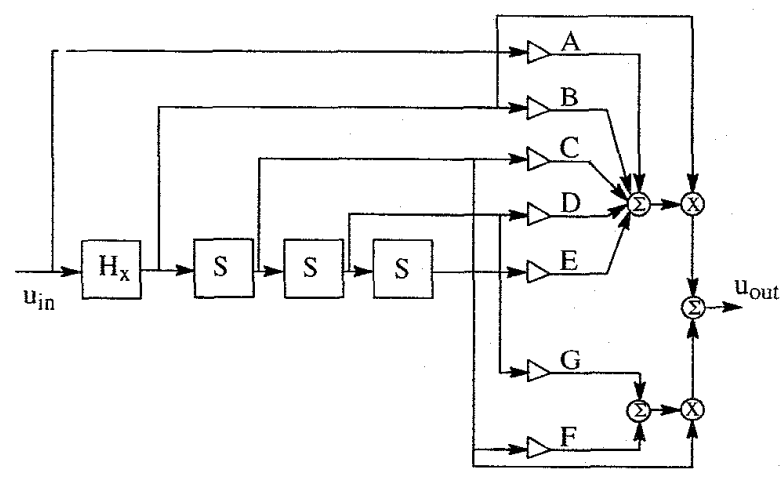

Figure 2: Second order compensator-2 according to Eq.(8).

area as depicted in Figure 3(b) and (c) for a sample frequercy of $15 \mathrm{kHz}$. Compared with a FIR realization based on the Chebyshev approximation [6] we would need at 
least an 8 tap FIR filter to obtain such a small error. Single disadvantage of the IIR realization is the fractional groupdelay of 0.58 sample. We therefore use a fractional sample delayer formed by a linear interpolator to obtain an equal delay in the parallel paths of the algorithm.

Before we test both algorithms on the real loudspeaker we take a closer look at the error in the amplitude of the output of both digital realizations caused by the linear filters. The error introduced by the bilinear transformation in the linear filter $H_{x}(z)$ is negligible small, and therefore major error in the output of the compensators is generated by the differentiators. As the error of the differentiator is known with respect to the frequency we are able to calculate the

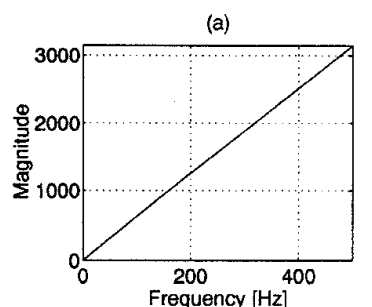

(c)

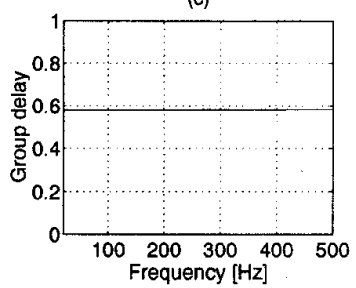

Figure 3: (a) Amplitude response of the used IIR differentiator $\left(T=1 / 15 \cdot 10^{3} \mathrm{sec}\right)$ and $(b)$ relative error with respect to frequency. (c) Group delay and (d) phase response show clearly the near linear phase properties for low frequencies.

propagation of this error through the algorithm. Results of simulations are given in Figure 4, where the relative error in the output of both compensators with respect to

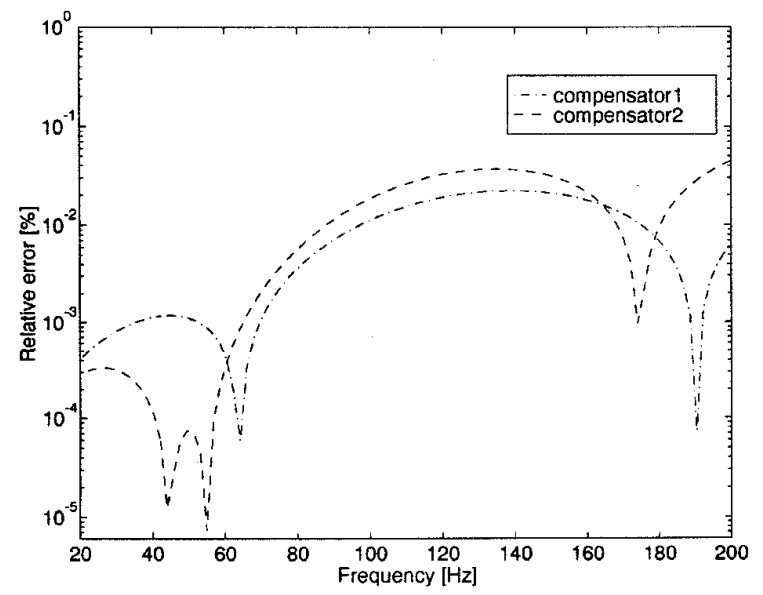

Figure 4: Relative error in the amplitude of the output of both second order kernels realizations with respect to the frequency.

the frequency are depicted. Clearly visible is that the error of compensator-2 is smaller for frequencies up to $60 \mathrm{~Hz}$ with a minimum around $50 \mathrm{~Hz}$. Above $60 \mathrm{~Hz}$ compensator-1 has a slightly better performance up to frequencies of $200 \mathrm{~Hz}$. As diaphragm excursions are inversively proportional to the square of frequency, distortions are at a maximum at the very low frequencies, in the range between 20 and $100 \mathrm{~Hz}$. It is therefore not interesting to consider the compensator performance for frequencies above $200 \mathrm{~Hz}$ as distortions are small above this frequency.

\section{Results}

Both compensation circuits are implemented in real-time on a TMS320C30 DSP which is mounted on a PC board. Using the high level design and simulation software SPW from Alta-group, C-Code is automatically generated from schematic entry of the algorithms.

Results of the compensators are measured at two different driving levels with a microphone in the near field. In Figure 5(a) and (b) we clearly see the agreement between the predicted error in Figure 4 and the performance of both compensators. Despite the difference between the compensators they both reduce second order distortion, where compensator- 2 yields best results with at most 20 percent reduction in the low frequency range.

The difference in the performance between the compensators is even more clear when the sound pressure level of the second order harmonic with respect to the driving frequency $f_{l}$ is considered, see Figure 6(a) and (b). The resemblance with the shape of the error curves in Figure 4 is
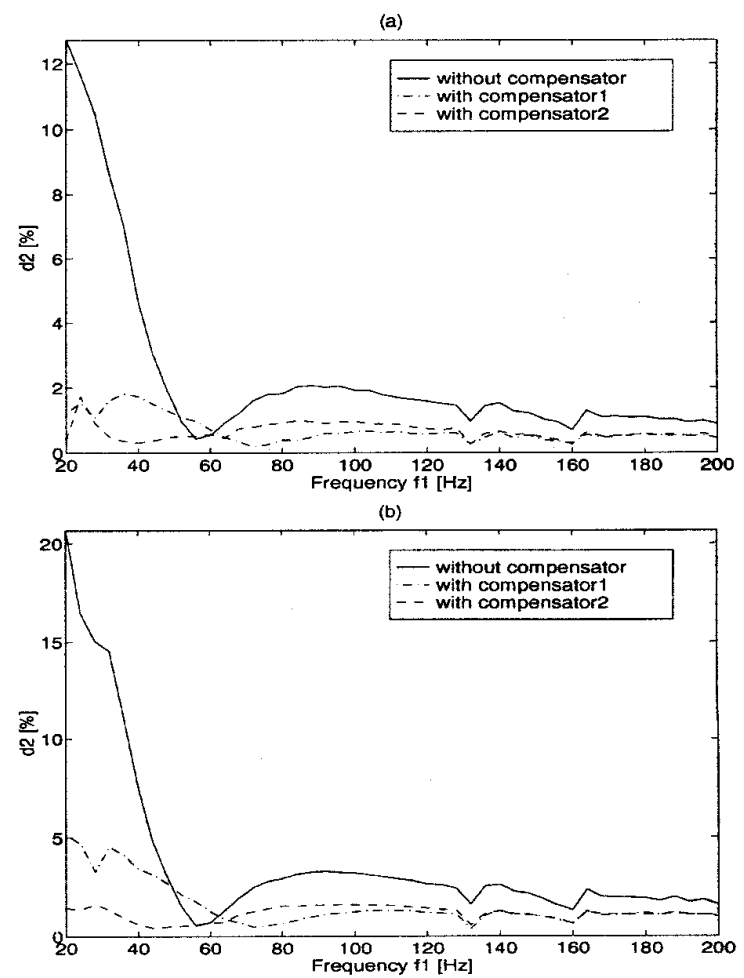

Figure 5: Measured relative second order harmonic distortion (d2) from the loudspeaker. Driving level at the loudspeaker terminals is $5 V_{\text {eff }}$ in $(a)$ and $7.5 V_{\text {eff }}$ in $(b)$. 

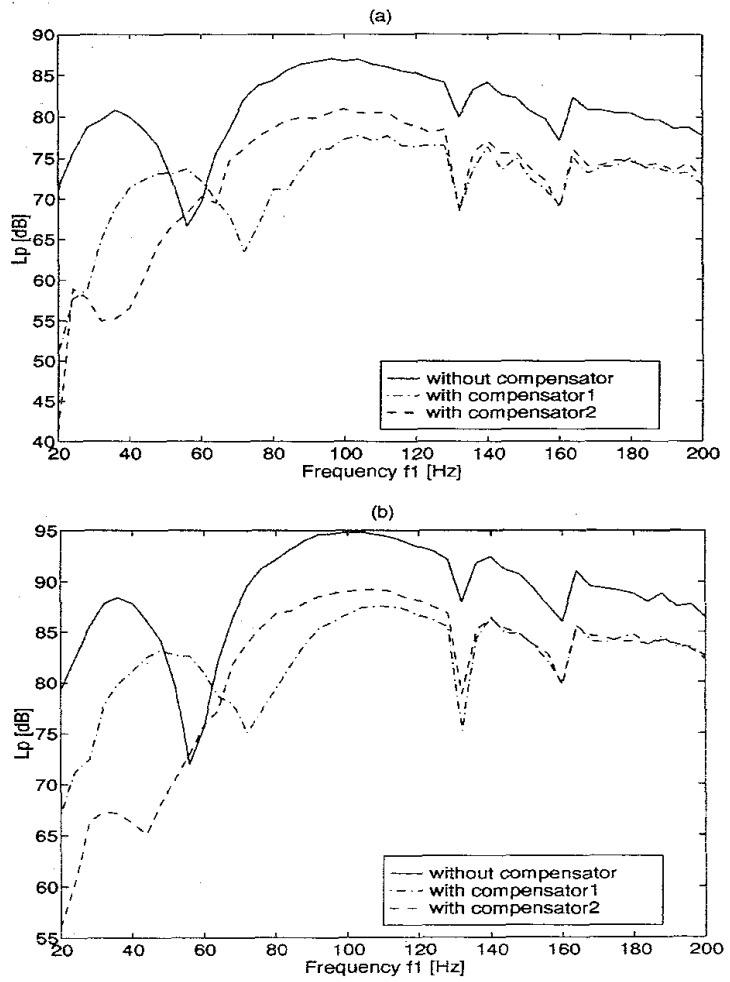

Figure 6: Measured sound pressure level $L_{p}\left(P_{r e f}=20 \mu \mathrm{Pa}\right)$ of the second harmonic. Driving level at the loudspeaker terminals is $5 V_{\text {eff }}$ in $(a)$ and $7.5 V_{\text {eff }}$ in $(b)$.

striking. For frequencies above $150 \mathrm{~Hz}$ the difference between both compensators vanishes. The dip in the second order harmonic between 55 and $60 \mathrm{~Hz}$ is caused by the mechanical resonance of the loudspeaker. At this frequency the current $i$ has a minimum value and therefore the force factor $B l(x)$ and self inductance $L_{e}(x)$ nonlinearities have a minimum influence around this frequency. Due to the greater error in compensator-1 compared to compensator-2 (see Figure 4), the second order harmonic is increased instead of reduced around $57 \mathrm{~Hz}$.

Performance of the reduction is independent of the driving level and third and higher order harmonics are not increased. Compared with previous results on reduction with Volterra compensators $[7,8]$, we have obtained a substantial better performance in a wider frequency span.

\section{Conclusions}

We have presented two different second order Volterra inverses to compensate for nonlinear behavior in electrodynamical loudspeakers. Both circuits are implemented as digital feedforward compensators and resulted in a significant reduction of second order harmonic distortion. The structure of compensator- 2 turned out to have the best performance, as could have been expected from the error curves of Figure 4 . In the market there is a growing need for small and cheap loudspeakers with good bass reproduction. The decreasing prizes of DSP hardware makes this compensator a viable solution to the design problem. Future work will concentrate on higher order compensation circuits and application to other transducers with strong nonlinear behavior, e.g. hom loudspeakers and hearing aid receivers. Major disadvantage of Volterra series as tool for modelling and compensation of system nonlinearities is the enormous increase in complexity when high(er) order systems are considered. Therefore other techniques to eliminate transducer nonlinearity will also be studied in the future.

\section{Acknowledgements}

The contribution of Mark A. Boer and Alex G.J. Nijmeijer to the research presented here is highly appreciated.

\section{References}

[1] A.J.M. Kaizer, On the design of broadband electrodynamical loudspeakers and multiway loudspeaker systems, Ph.D. thesis Technical University of Eindhoven, 1986

[2] H.F. Olsen, Acoustical engineering, Van Nostrand, New York, 1967

[3] J.A. Melder and R. Nead, "The convergence of a class of double-rank minimization algorithms," J. Inst. Math. Applic., Vol. 6, pp. 76-90, 1970

[4] M. Schetzen, The Volterra and Wiener theories of nonlinear systems, John Wiley, New York, 1980

[5] M.A. Al-Alaoui, "Novel IIR differentiator from the Simpson integration rule," IEEE transactions on circuits and systems-1: Fundamental theory and applications, vol.41, pp. 186-187, February 1994

[6] T.W. Parks and J.H. McClellan, "Chebyshev approximation for nonrecursive digital filters with linear phase," IEEE Trans. on Circuit Theory, vol. CT-19, pp. 189-194, Mar:h 1972

[7] R. de Vries, A.P. Berkhoff, C.H. Slump, O.E. Herrmann, "Digital compensation of nonlinear distortion in loudspeakers," Proc. ICASSP-93, vol. I pp. 165-168, April 27-30 Minneapolis, USA, 1993

[8] H. Schurer, C.H. Slump and O.E. Herrmann, "Digital compensation of nonlinear distortion in loudspeakers," Proc. ProRISC/IEEE Benelux workshop on Circuits, Systems and Signal Processing, pp. 277-284, March 23\&24, Mierlo, The Netherlands, 1995 